\title{
SOME FEATURES OF SUPERCONDUCTING DUAL BORE LENS*
}

\author{
A. A. Mikhailichenko \\ Wilson Laboratory, Cornell University, Ithaca, NY $14850^{\dagger}$
}

\begin{abstract}
Design of dual bore superconducting quadrupole for CESR upgrade is represented. The lens has a gradient about $1 \mathrm{kG} / \mathrm{cm}$, dual aperture of $54 \mathrm{~mm}$ each, length about $800 \mathrm{~mm}$, The axes spacing about $80 \mathrm{~mm}$ allows a good separation of counter rotating beams. Harmonics content measured below $0.05 \%$. Some concepts used in this dual bore lens could be used for design of any multi aperture lens, where the high field quality required.
\end{abstract}

\section{INTRODUCTION}

Cornell storage ring CESR finished Stage II upgrade, achieved highest luminosity what exceeds now $L \cong 7 \cdot 10^{32} \mathrm{~cm}^{-2} \mathrm{~s}^{-1}$. After the Stage III is finished, the luminosity will increase up to $3 \cdot 10^{33} \mathrm{~cm}^{-2} \mathrm{~s}^{-1}$. More drastic increase is expecting after Stage IV is finished [1-3], Fig. 1. This Stage when completed will allow to have at least 180 counter rotating bunches $3 \times 3 \mathrm{~A}^{2}$ total in individual vacuum chambers. The beams having equal energies will deliver a $25 \mathrm{fb}^{-1}$ month ${ }^{-1}$ with $L \cong 3 \cdot 10^{34} \mathrm{~cm}^{-2} \mathrm{~s}^{-1}$. The vacuum chambers will share wide aperture room temperature dipole magnets and will have dual bore superconducting magnets. These superconducting magnets include dual bore quadrupole, dual bore sextupole, and dual bore dipole correctors. Also some dual bore octupoles or skew quadrupoles will be used instead of dipole correctors. All three types of these magnets will share the same cryostat and considered as one unit. About 88 such units together with 156 regular arc dipoles will be installed on the top of existing synchrotron, Fig.1. These new rings will be feed directly from this synchrotron, allowing CESR as a dedicated synchrotron source.

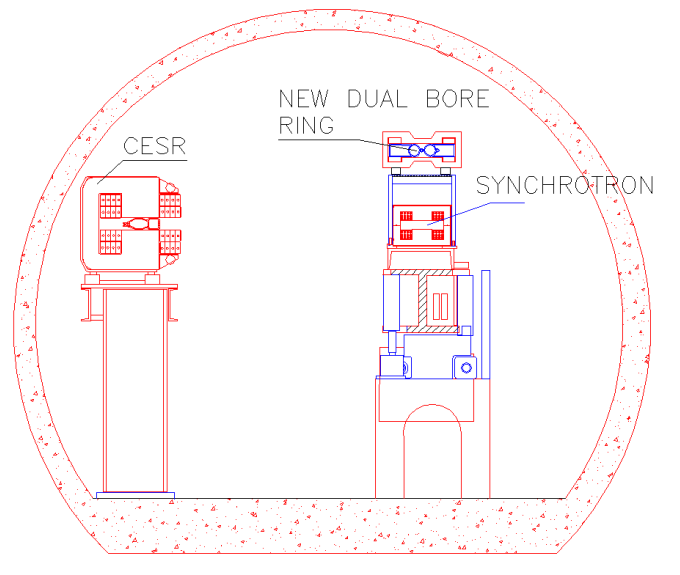

Figure 1: The tunnel cross-section.
Utilization of CESR as a booster between synchrotron and this new dual bore machine could bring the luminosity further up to $L \geq 10^{35} \mathrm{~cm}^{-2} \mathrm{~s}^{-1}$ level.

Extreme beam parameters yield extreme requirements to the magnet and vacuum system design. These requirements are the field quality, ability to work under extreme expose to SR and closest position of the beams axes. The last required for minimal dipole magnet crosssection.

In this publication we describe a dual bore quadrupole lens design and results of its test. This quadrupole magnet itself was tested in a Dewar up to 200 A feeding current $[2,5]$. The test showed that the field accuracy required has been achieved [5]. Now mostly attention was paid to a cryostat, holding the cold mass with dual bore magnets. The vacuum chamber of superconducting magnet must hold about $2 \mathrm{~kW} / \mathrm{m}$ of SR power in normal operation. This fact strongly complicated the design.

For normal operation the number of current leads from room temperature to the cold mass is twelve per magnet unit. Utilization of High Temperature Superconductor (HTS) leads is crucial here. We are planning to compare the HTS leads manufactured by Hoechst Company and American Superconductor.

Results obtained in the test described will be used for a final magnet design.

\section{QUADRUPOLE CONCEPT}

Each of quadrupoles is a combination of two quadrupole magnets that share the same iron yoke. Axes separation needs to be as small as possible. Technical limitations make this distance about $80 \mathrm{~mm}$. The field quality required for the quadrupole is about $\left[B_{y}(x)-G \cdot x\right] / G \cdot x \leq 5 \cdot 10^{-4}$, where $B_{y}(x)$ is the field across the aperture, $G$ is a gradient. When dual bore lenses shared the same yoke and placed close one to another, some magnetic lines travel around both centers, Fig.2. So in the presence of neighboring lens, magnetic lines lose its quadrupole symmetry in the yoke, and, hence in all lens. Now not only quadrupole-associated harmonics allowed. Dipole and sextupole components are the strongest among allowed now. The same happens, sequentially, for the whole lens. Proper modeling includes the lowest symmetry piece taking into account this effect [5]. So one needs to have ability for effective compensation of this effect. This mechanism needs to be simple and effective. The lenses, where the poles and the coils acting together for the field generation were chosen finally for the next

\footnotetext{
* Work supported by National Science Foundation

† Phone: (607) 255-3785, Fax: (607) 255-8062, e-mail: mikhail@lns62.lns.cornell.edu
} 
stage of CESR upgrade. This type of lens was suggested in [4]. The superconducting coils used there are a racetrack type of single layer windings.

For the requirements of the beam optics the gradients in both neighboring lenses have the same sign and about the same value. So the neighboring currents on the sides of the septum have the same value. The last yields that vertical component of magnetic induction in the iron septum is absent practically.

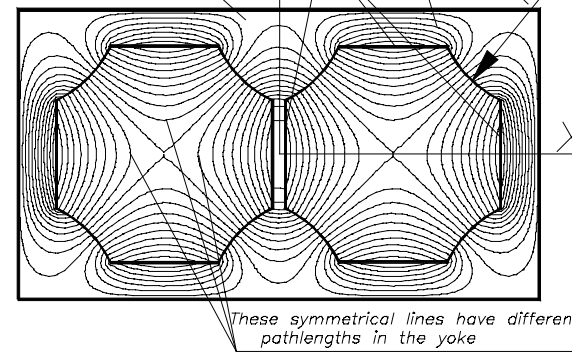

Figure 2: Magnetic lines in dual bore lens. Windings are single layer racetrack coils, which cross-sections are thin in the drawing's scale. They occupy the whole straight sides. The gradients in both lenses have the same sign. The distance between quadrupole axes is $79 \mathrm{~mm}$. Yoke septum is $4 \mathrm{~mm}$. Printout from MERMAID [6]

Similar behavior has any dual bore symmetrical lens. One can also see from Fig.2, that the lines spaced at the same distance from the center of each quad have different path lengths in the material of yoke. The shortest ways have the lines that travel around currents in the center of the lens. So the region marked as $x-y$ quadrant is minimal for proper modeling.

To compensate this effect, some appropriate nonsymmetrical deformation of the poles required. Calculations show, that the accuracy of fabrication must be $\delta \approx 8.5 \cdot 10^{-3} \mathrm{~mm}$. One can see that the profile is rather complicated. In simplest case, however, when an arc of constant radius approximates hyperbola, the arc radius of the left and right poles could be made slightly different, keeping the vertex of the arcs in the same place. We keep the radiuses of all poles the same, but different from one, obtained, when one lens is far apart from neighboring one. Below we represent the graphs of absolute deviation of the field for different pole radius. Absolute value of gradient is also slightly different in each case. The smallest deviation from linear law indicates the lens with the pole radius of $37.45 \mathrm{~mm}$. Maximum deviation of 0.15 Gauss occurs at distance $2.25 \mathrm{~cm}$. Magnetic field value, associated with pure quadrupole having gradient $G=1.075 \mathrm{kG} / \mathrm{cm}$ (for this particular radius and for 6 $\mathrm{kA} /$ coil) is $2.4187 \mathrm{kG}$. So, relative deviation in this point is $0.15 / 2418.7=6.2 \cdot 10^{-5}$. Namely this radius was chosen for manufacturing.

At the end of the lens some soft iron shields made. They prevent the field interference from neighboring lenses.

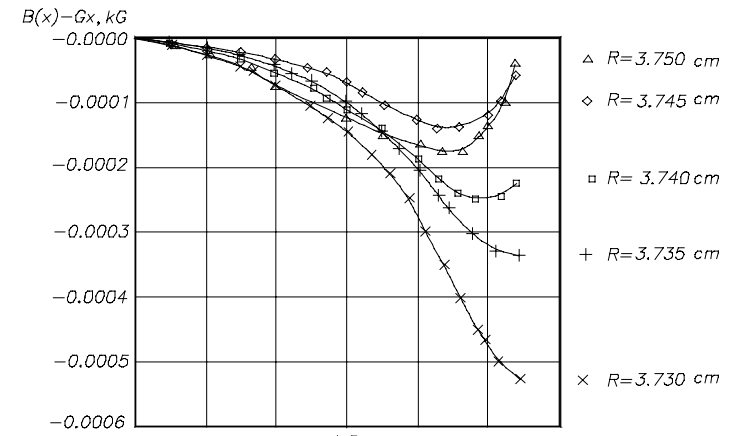

Figure 3: Calculated field deviation (in $k G$ ) from linear behavior for different pole radius ( $\mathrm{R}$ in Fig.2).

The lens was fabricated and tested. Results of measurements are represented in Fig. 4.

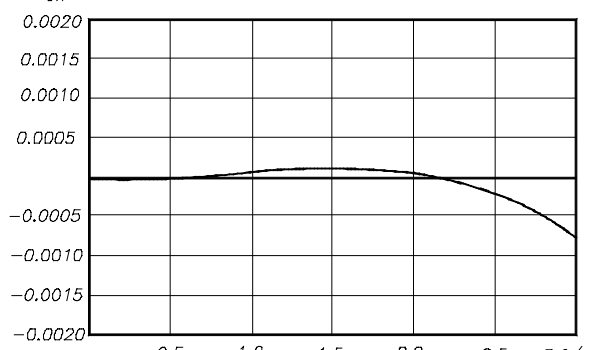

Figure 4: Measured relative field deviation in one of the dual bore aperture. $G=1.075 \mathrm{kG} / \mathrm{cm}$.

The graph plotted is a reconstruction of a vector sum of different harmonics measured in assembled dual bore magnet. These measurements were carried out in a Dewar. Warm long coil was rotated inside the space arranged with two coaxial stainless steel tubes going through the magnet, see [2].

\section{CRYOSTAT}

Cryostat serves as a vessel for liquid Helium with cold mass in it. Supposed that the Helium/Nitrogen duct running along the ring will have the branches to each magnet. The cryostat concept is shown in Fig. 5. Mostly crowded place here is inner region. SR is accepted by the inner chamber made on two coaxial copper tubes having the gap about $1.5 \mathrm{~mm}$ for the water flow in between. The thickness of the copper tubes is about $1.5 \mathrm{~mm}$ also. The inner chamber is made removable. This inner chamber wrapped by superinsulation. The next $1.5 \mathrm{~mm}$ thick tube is a part of a Nitrogen shield and also wrapped by superinsulation. Cooling of these last tubes is going through the ends. The vacuum chamber of the ring and the vacuum volume inside the cryostat are independent. The next tube is a part of Helium vessel. All tubes are polished to a mirror shine. Design of supports (hatched in Fig.5) allows shrinkage of the length keeping the central part of quadrupole in fixed position. It allows positioning of cold mass and Nitrogen shield in the cabinet also. HTS leads at cold end bolted to feeds- through into liquid 
Helium vessel and at the other side bolted to the Nitrogen shield with thing layer of mica for electrical insulation.
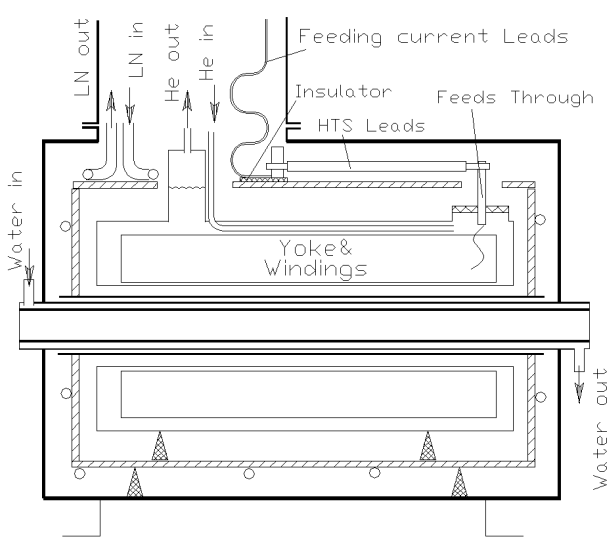

Figure 5: Basic principles of cryostat design.

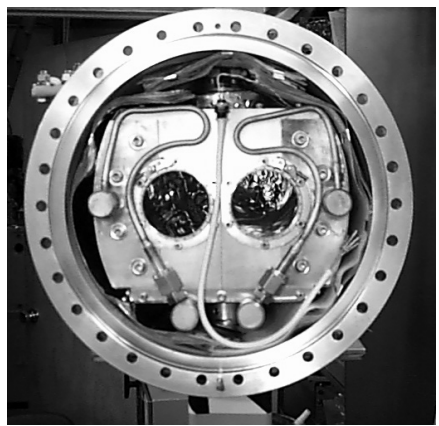

Figure 6: Inside view to the cryostat. Here one can see a Nitrogen temperature shield with tubing. End flange and superinsulation removed. Inner diameter is $\cong 10$ inches. One can see here two independent apertures.

The cryogenic test proved the principles. The magnet was attached to the existing system for Superconducting RF cavity feed. This system includes both liquid Helium and Nitrogen tubing. The necessary cooling was achieved. Vacuum inside the cryostat goes to $2.810^{-8}$ Torr as a result of cryocooling. In this test the HTS leads made by American Superconductor were used. These leads are 16" long and must have a critical current of 150 A. The feeding of the lens with current showed however, that the current of 25 A yields a loss of HTS ability to carry the current. This was explained by inadequate cooling of HTS leads. The latest modification done includes the increase of cooling of HTS leads. Also done some improvements in pressure relief system, in monitoring the pressure inside the cold mass, in measuring the temperature of cold mass and Nitrogen cooled shield. In nearest future we will continue the test of the cryostat. Modeling of SR deposition will be done with a special heater located inside the vacuum chamber.

\section{CONCLUSION}

The nonlinarities arisen from broken quadrupole symmetry into iron can be taken into account by proper choice of problem for modeling. The set of tests done proved the principles of design made. More attention to the proper cooling of HTS leads was stressed.

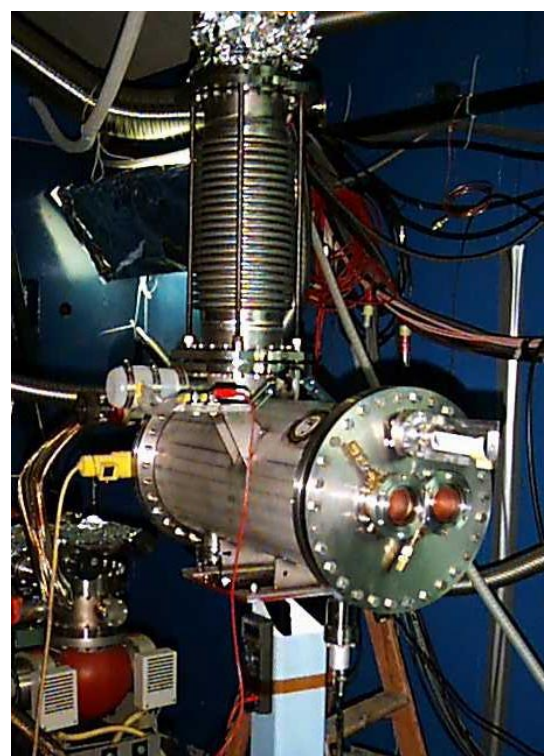

Figure 7: Dual bore magnet installed in a cryostat for cryogenic test. In a vertical bellow there are joints to existing cryogenic system. The joints to a duct line running along the ring will be located there in a future.

The same ideas of cancellation interference were applied to other dual bore multipole elements (such as sextupole, skew quadrupole, octupole and dipole corrector) of the ring. Serially installed quads and sextupoles in each module also may give the way for adjustment the resulting field quality for whole unit.

Mostly strong interference occurs at the magnet dual edge. Solution of this problem in application to the dual bore magnet was found and proved experimentally in this design.

\section{REFERENCES}

[1] D. Rubin, M. Tigner, Shared bends and Independent Quadrupoles, Cornell CON 94-28, 1994.

[2] A. Mikhailichenko, D. Rubin, Concentric Ring Colliding Beam Machine with Dual Aperture Quadrupoles, Cornell CLNS 96-1420, 1996.

[3] G. Dugan, A.A.Mikhailichenko, J. Rojers, D. Rubin, Dual Aperture High Luminosity Collider at Cornell, Talk on Particle Accelerator Conference PAC 97, Vancouver, B.C. Canada, 12-17 May 1997, 6B10. Proceedings, p. 318.

[4] A. Mikhailichenko, 3D Electromagnetic Field. Representation and measurement, CBN 95-16, Cornell, 1995.

[5] A. A. Mikhailichenko, Some peculiarities of Magnetic Field Behavior in Dual Bore Magnets, CBN 97-32, October 17, 1997, Cornell.

[6] MERMAID --MEsh oriented Routine for MAgnet Interactive Design, SIM Limited, Novosibirsk, P.O. Box 402, Russia. 